
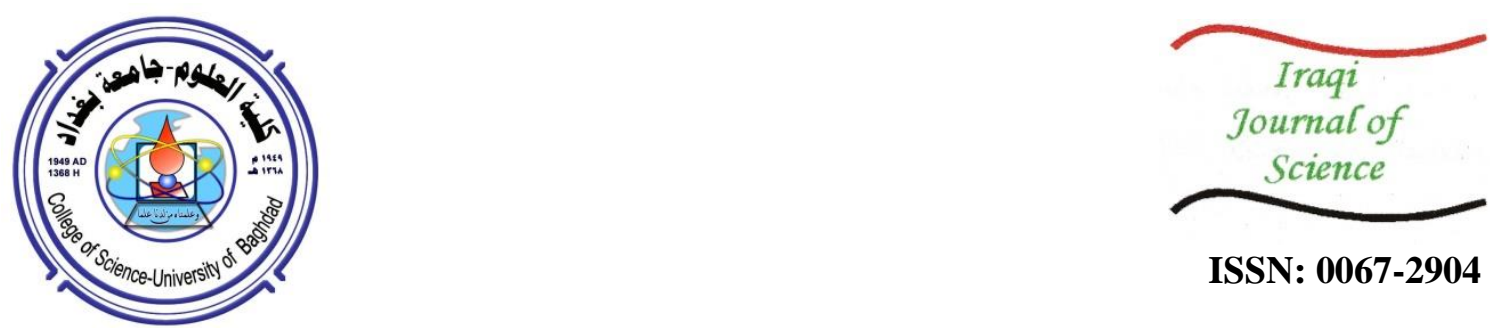

ISSN: 0067-2904

\title{
Some Properties of D-Operator on Hilbert Space
}

\author{
Eiman H. Abood*1, Samira N. Kadhim²
}

${ }^{1}$ Department of Mathematics, College of Science, University of Baghdad, Baghdad, Baghdad, Iraq ${ }^{2}$ Department of Mathematics, College of Science for Women, University of Baghdad, Baghdad, Iraq

Received: $27 / 6 / 2020$

Accepted: 10/10/2020

\begin{abstract}
In this paper, we introduce a new type of Drazin invertible operator on Hilbert spaces, which is called D-operator. Then, some properties of the class of Doperators are studied. We prove that the D-operator preserves the scalar product, the unitary equivalent property, the product and sum of two D-operators are not Doperator in general but the direct product and tenser product is also D-operator.
\end{abstract}

Keywords: Hilbert space, Drazin operator, Normal operator, D-normal operator, nnormal operator, class (Q) operators.

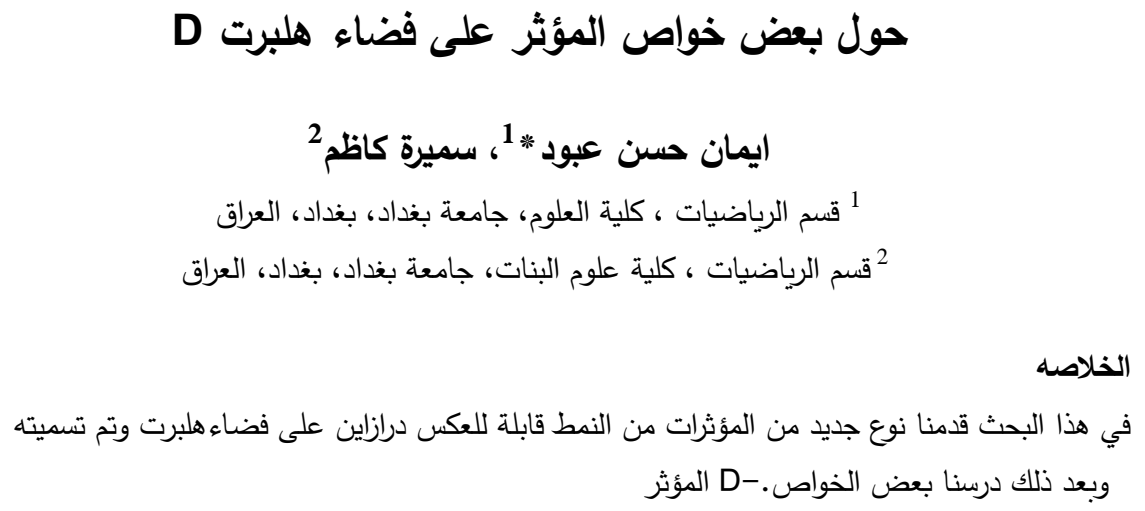

\section{Introduction}

Throughout this paper, $H$ is a Hilbert space, $B(H)$ is the space of all bounded linear operators on a complex Hilbert space $H$. The Drazin inverse for a bounded linear operator on a complex Banach space was introduced by Caradus [1]. Let $T \in B(H)$, the Drazin inverse of $T$, if it exists, is an operator $T^{D} \in B(H)$ such that

$T T^{D}=T^{D} T, T^{D} T T^{D}=T^{D}, T^{k+1} T^{D}=T^{k}$

For some integer number $k \geq 0$, the smallest integer $k \geq 0$ is called the index of $T$ which is denoted by ind $(T)$. It is easy to see that ind $(T)=0$ if and only if $T$ is an invertible operator. Then $T^{D}=T^{-1}$. In the following lemma, we collect some properties of Drazin operator which appeared in previous studies [2,3].

Lemma 1.1: Let $S, T \in B(H)$ be two Drazin invertible operators, then

(a) $\quad\left(T^{*}\right)^{D}=\left(T^{D}\right)^{*}$.

(b) $\quad\left(T^{\ell}\right)^{D}=\left(T^{D}\right)^{\ell}$ for $\ell=1,2, \ldots$

(c) $\quad\left(S^{-1} T S\right)^{D}=S^{-1} T^{D} S$.

(d) If $S T=T S$, then $(S T)^{D}=S^{D} T^{D}=T^{D} S^{D}, S^{D} T=T S^{D}$, and $T^{D} S=S T^{D}$.

(e) If $S T=T S=0$, then $(S+T)^{D}=S^{D}+T^{D}$. 
Let $T \in B(H), T$ is called normal if $T T^{*}=T^{*} T$. The purpose of this paper is to introduce a new operator to generalize the normal operator. Many authors presented generalizations of normal operators. For examples, in an earlier work [4], the author introduced a class $(Q)$ of operators acting on a Hilbert space $\mathrm{H}$ : for any $T \in(Q), T^{* 2}(T)^{2}=\left(T^{*} T\right)^{2}$. Then, in another article [5], the authors introduced some new classes of operators associated with Drazin invertible operator. In this section, we give a new type of operators that are associated with Drazin invertible operator, that we call Doperator.

The paper contains two sections. In section one, we investigate some basic properties that we need. In section two, we study most of the properties of D-operators.

\section{Main Results \\ Definition 2.1:}

Let $T \in B(H)$ be Drazin invertible. $T$ is called D-operator if $T^{* 2}\left(T^{D}\right)^{2}=\left(T^{*} T^{D}\right)^{2}$.

The class of all D-operators is denoted by [D]. By lemma (1.1) (d), it is easy to prove that every normal operator is D-operator but the converse is not true in general. For example, let $T$ be a nilpotent operator, then $T^{D}=0$, hence it is clear that $T$ is D-operator but the nilpotent is not necessary normal.

In this section, we investigate some basic properties of operators in [D].

\section{Proposition 2.2:}

Let $T \in[D]$, then the following assumptions hold:

1) $\alpha T \in[D]$ for every scalar $\alpha$.

2) $S \in[D]$ for every $S \in B(H)$ that is unitary equivalent to $T$.

3) The restriction $T \mid M$ of $T$ to any closed subspace $M$ of $H$ that reduces $T$ is in [D].

4) $T^{D} \in[D]$.

\section{Proof:}

1) The proof is straight forward.

2) Since $\mathrm{S}$ is unitary equivalent to $\mathrm{T}$, then $S=U T U^{*}$, where $U$ is unitary operator. Thus, $S^{* 2}\left(S^{D}\right)^{2}=\left(U T^{*} U^{*}\right)^{2}\left(U T^{D} U^{*}\right)^{2}$

$$
\begin{aligned}
& =\left(U T^{*} U^{*}\right)\left(U T^{*} U^{*}\right)\left(U T^{D} U^{*}\right)\left(U T^{D} U^{*}\right) \quad\left(U U^{*}=I\right) \\
& =U T^{*} T^{*} T^{D} T^{D} U^{*} \\
& =U T^{* 2}\left(T^{D}\right)^{2} U^{*} \\
& =U\left(T^{*} T^{D}\right)^{2} U^{*} \\
& =U T^{*} T^{D} T^{*} T^{D} U^{*} \\
& =\left(U T^{*} U^{*}\right)\left(U T^{D} U^{*}\right)\left(U T^{*} U^{*}\right)\left(U T^{D} U^{*}\right) \\
& =S^{*} S^{D} S^{*} S^{D} \\
& =\left(S^{*} S^{D}\right)^{2}
\end{aligned}
$$

Hence, $S \in[D]$.

3) $\quad(T \mid M)^{* 2}\left((T \mid M)^{D}\right)^{2}=(T \mid M)^{*}(T \mid M)^{*}(T \mid M)^{D}(T \mid M)^{D}$

$$
\begin{gathered}
=\left(T^{*} \mid M\right)\left(T^{*} \mid M\right)\left(T^{D} \mid M\right)\left(T^{D} \mid M\right) \\
=\left(T^{*} T^{*} \mid M\right)\left(T^{D} T^{D} \mid M\right) \\
=\left(T^{* 2} \mid M\right)\left(\left(T^{D}\right)^{2} \mid M\right) \\
=\left(T^{* 2}\left(\mathrm{~T}^{\mathrm{D}}\right)^{2}\right) \mid \mathrm{M} \\
=\left(\left(T^{*} T^{D}\right)^{2}\right) \mid M \\
=\left(\left(T^{*} T^{D}\right)\left(T^{*} T^{D}\right)\right) \mid M \\
=\left(\left(\left(T^{*} T^{D}\right) \mid M\right)\left(\left(T^{*} T^{D}\right)\right) \mid M\right) \\
=\left(\left(T^{*} \mid M\right)\left(T^{D} \mid M\right)\left(T^{*} \mid M\right)\left(T^{D} \mid M\right)\right) \\
=\left(\left(T^{*} \mid M\right)\left(T^{D} \mid M\right)\right)^{2} \\
=\left((T \mid M)^{*}(T \mid M)^{D}\right)^{2} .
\end{gathered}
$$

Hence $T \mid M \in[D]$.

4) Since $T \in[D]$, then $\quad T^{* 2}\left(T^{D}\right)^{2}=\left(T^{*} T^{D}\right)^{2}$. Thus $T^{*} T^{*} T^{D} T^{D}=T^{*} T^{D} T^{*} T^{D}$

By taking the adjoint of both sides of the above equation, we have

Hence,

$$
\left(T^{*}\right)^{D}\left(T^{*}\right)^{D} T T=\left(T^{*}\right)^{D} T\left(T^{*}\right)^{D} T
$$

$$
\left(\left(T^{D}\right)^{*}\right)^{2} T^{2}=\left(\left(T^{D}\right)^{*} T\right)^{2} \text {. }
$$


Therefore,

Thus, $T^{D} \in[D]$

$$
\left(\left(T^{D}\right)^{*}\right)^{2}\left(\left(T^{D}\right)^{D}\right)^{2}=\left(\left(T^{D}\right)^{*}\left(T^{D}\right)^{D}\right)^{2}
$$

\section{Proposition 2.3:}

The set of all D-operators on $H$ is a closed subset of $B(H)$.

\section{Proof:}

Let $\left\langle T_{k}\right\rangle$ be a sequence of D-operators such that $T_{k} \rightarrow T$. It is enough to show that $T$ is D-operator.

Since $T_{k} \rightarrow T$ then $T_{k}{ }^{*} \rightarrow T^{*}$ and $T_{k}{ }^{D} \rightarrow T^{D}$. Hence, $T_{k}{ }^{*} T_{k}{ }^{D} \rightarrow T^{*} T^{D}$, then we get that

$$
\left(T_{k}{ }^{*} T_{k}{ }^{D}\right)^{2} \rightarrow\left(T^{*} T^{D}\right)^{2}
$$

On the other hand, we obtain that $T_{k}^{* 2} \rightarrow T^{* 2}$ and $\left(T_{k}{ }^{D}\right)^{2} \rightarrow\left(T^{D}\right)^{2}$. Hence, $T_{k}^{* 2}\left(T_{k}{ }^{D}\right)^{2} \rightarrow$ $T^{* 2}\left(T^{D}\right)^{2}$

Therefore, from equations (1) and (2), we conclude that

$$
\begin{aligned}
\left\|T^{* 2}\left(T^{D}\right)^{2}-\left(T^{*} T^{D}\right)^{2}\right\| & \\
& =\left\|T^{* 2}\left(T^{D}\right)^{2}-T_{k}^{* 2}\left(T_{k}^{D}\right)^{2}+T_{k}^{* 2}\left(T_{k}^{D}\right)^{2}-\left(T^{*} T^{D}\right)^{2}\right\| \\
& \leq\left\|T^{* 2}\left(T^{D}\right)^{2}-T_{k}^{* 2}\left(T_{k}^{D}\right)^{2}\right\|+\left\|T_{k}^{* 2}\left(T_{k}^{D}\right)^{2}-\left(T^{*} T^{D}\right)^{2}\right\| \\
& =\left\|T^{* 2}\left(T^{D}\right)^{2}-T_{k}^{* 2}\left(T_{k}^{D}\right)^{2}\right\|+\left\|\left(T_{k}^{*} T_{k}^{D}\right)^{2}-\left(T^{*} T^{D}\right)^{2}\right\|
\end{aligned}
$$

$k \rightarrow \infty$.

Hence, $T^{* 2}\left(T^{D}\right)^{2}=\left(T^{*} T^{D}\right)^{2}$. Thus $T \in[D]$

\section{Proposition 2.4:}

Let $S, T \in[D]$. If $[T, S]=\left[T, S^{*}\right]=0$, then $S T \in[D]$.

\section{Proof:}

Since $[T, S]=\left[T, S^{*}\right]=0$, then by lemma(1.1) (d) we have $\left[T, S^{D}\right]=\left[T^{D}, S\right]=\left[T^{D}, S^{*}\right]=\left[T^{*}, S^{D}\right]=0$.

Moreover, since $S, T \in[D]$, then

$$
\begin{aligned}
& T^{* 2}\left(T^{D}\right)^{2}=\left(T^{*} T^{D}\right)^{2} \text { and } S^{* 2}\left(S^{D}\right)^{2}=\left(S^{*} S^{D}\right)^{2} \text {. Therefore, } \\
& (S T)^{* 2}\left((S T)^{D}\right)^{2}=(S T)^{*}(S T)^{*}(S T)^{D}(S T)^{D} \\
& =T^{*} S^{*} T^{*} S^{*} S^{D} T^{D} S^{D} T^{D} \\
& =T^{*} T^{*} T^{D} T^{D} S^{*} S^{*} S^{D} S^{D} \\
& =T^{* 2} S^{* 2}\left(T^{D}\right)^{2}\left(S^{D}\right)^{2} \\
& =T^{*} T^{*} S^{*} S^{*} T^{D} S^{D} T^{D} S^{D} \\
& =T^{*} S^{*} T^{*} S^{*} T^{D} S^{D} T^{D} S^{D} \\
& =(S T)^{*}(S T)^{*}(S T)^{D}(S T)^{D} \\
& =\left((S T)^{*}(S T)^{D}\right)^{2}
\end{aligned}
$$

Thus $S T \in[D]$

\section{Proposition 2.5:}

\section{Proof:}

Let $S, T \in[D]$. If $S T=T S=0$, then $S+T \in[D]$.

(f) Since $S, T \in[D]$, then $T^{* 2}\left(T^{D}\right)^{2}=\left(T^{*} T^{D}\right)^{2}$ and $S^{* 2}\left(S^{D}\right)^{2}=\left(S^{*} S^{D}\right)^{2}$. Since $S T=T S=0$, then $S^{*} T^{*}=T^{*} S^{*}=0$, and by lemma $(1.1)(\mathrm{e})$ we have $(S+T)^{D}=S^{D}+T^{D}$. Hence

$(S+T)^{* 2}\left((S+T)^{D}\right)^{2}=(S+T)^{*}(S+T)^{*}(S+T)^{D}(S+T)^{D}$

$$
\begin{aligned}
& =\left(S^{*}+T^{*}\right)\left(S^{*}+T^{*}\right)\left(S^{D}+T^{D}\right)\left(S^{D}+T^{D}\right) \\
& =\left(S^{* 2}+T^{* 2}\right)\left(\left(S^{D}\right)^{2}+\left(T^{D}\right)^{2}\right) \\
& =S^{* 2}\left(S^{D}\right)^{2}+T^{* 2}\left(T^{D}\right)^{2} \\
& =\left(S^{*} S^{D}\right)^{* 2}+\left(T^{*} T^{D}\right)^{2} \\
& =\left(S^{*} S^{D}+T^{*} T^{D}\right)\left(S^{*} S^{D}+T^{*} T^{D}\right) \\
& =\left(S^{*}+T^{*}\right)\left(S^{D}+T^{D}\right)\left(S^{*}+T^{*}\right)\left(S^{D}+T^{D}\right) \\
& =\left((S+T)^{*}(S+T)^{D}\right)^{2}
\end{aligned}
$$

Thus $S+T \in[D]$

The following example shows that the propositions (2.4) and (2.5) are not necessarily true in general.

\section{Example 2.6:}


1) Let $S=\left(\begin{array}{ll}2 & 1 \\ 1 & 1\end{array}\right)$ and $T=\left(\begin{array}{ll}1 & 0 \\ 0 & 2\end{array}\right)$.

Therefore,

$$
S^{D}=\left(\begin{array}{cc}
1 & -1 \\
-1 & 2
\end{array}\right) \text { and } T^{D}=\left(\begin{array}{cc}
1 & 0 \\
0 & 1 / 2
\end{array}\right) \text {. }
$$

It can be easily checked that $S, T \in[D]$ and $S T \neq T S$. Note that,

$$
(S T)^{D}=\left(\begin{array}{cc}
2 & -1 \\
-1 & 2
\end{array}\right)
$$

But, it is easy to compute that $S T \notin[D]$.

2) Let $T=\left(\begin{array}{ll}1 & 0 \\ 0 & 2\end{array}\right)$. Clearly $T \in[D]$, but $T+I \notin[D]$

The following corollary is a straightforward result from proportion (2.4).

\section{Corollary 2.7:}

If $T \in[D]$, then $T^{n} \in[D]$ for all positive integers $n$.

\section{Theorem 2.8:}

Let $T_{1}, T_{2}, \ldots, T_{n} \in[D]$, then

1) $\quad T_{1} \oplus T_{2} \oplus \ldots \oplus T_{n} \in[D]$.

2) $\quad T_{1} \otimes T_{2} \otimes \ldots \otimes T_{n} \in[D]$.

\section{Proof:}

1) Since $T_{i} \in[D] \forall i=1,2, \ldots, n$, then

$$
\begin{aligned}
& T_{i}^{* 2}\left(T_{i}^{D}\right)^{2}=\left(T_{i}^{*} T_{i}^{D}\right)^{2} \text {. Hence, } \\
& \left(T_{1} \oplus T_{2} \oplus \ldots \oplus T_{n}\right)^{* 2}\left(\left(T_{1} \oplus T_{2} \oplus \ldots \oplus T_{n}\right)^{D}\right)^{2} \\
& =\left(T_{1}^{* 2} \oplus T_{2}^{* 2} \oplus \ldots \oplus T_{n}^{* 2}\right)\left(\left(T_{1}^{D}\right)^{2} \oplus\left(T_{2}^{D}\right)^{2} \oplus \ldots \oplus\left(T_{n}^{D}\right)^{2}\right) \\
& =T_{1}^{* 2}\left(T_{1}^{D}\right)^{2} \oplus T_{2}^{* 2}\left(T_{2}^{D}\right)^{2} \oplus \ldots \oplus T_{n}^{* 2}\left(T_{n}^{D}\right)^{2} \\
& =\left(T_{1}^{*} T_{1}^{D}\right)^{2} \oplus\left(T_{2}^{*} T_{2}^{D}\right)^{2} \oplus \ldots \oplus\left(T_{n}^{*} T_{n}^{D}\right)^{2} \\
& =T_{1}^{*} T_{1}^{D} T_{1}^{*} T_{1}^{D} \oplus T_{2}^{*} T_{2}^{D} T_{2}^{*} T_{2}^{D} \oplus \ldots \oplus T_{n}^{*} T_{n}^{D} T_{n}^{*} T_{n}^{D} \\
& =\left(T_{1}^{*} T_{1}^{D} \oplus T_{2}^{*} T_{2}^{D} \oplus \ldots \oplus T_{n}^{*} T_{n}^{D}\right)\left(T_{1}^{*} T_{1}^{D} \oplus T_{2}^{*} T_{2}^{D} \oplus \ldots \oplus T_{n}^{*} T_{n}^{D}\right) \\
& =\left(\left(T_{1}^{*} \oplus T_{2}^{*} \oplus \ldots \oplus T_{n}^{*}\right)\left(T_{1}^{D} \oplus T_{2}^{D} \oplus \ldots \oplus T_{n}^{D}\right)\right)^{2} \\
& =\left(\left(T_{1} \oplus T_{2} \oplus \ldots \oplus T_{n}\right)^{*}\left(T_{1} \oplus T_{2} \oplus \ldots \oplus T_{n}\right)^{D}\right)^{2}
\end{aligned}
$$

2) Let $x_{1}, x_{2}, \ldots, x_{n} \in H$, then

$$
\left(T_{1} \otimes T_{2} \otimes \ldots \otimes T_{n}\right)^{* 2}\left(\left(T_{1} \otimes T_{2} \otimes \ldots \otimes T_{n}\right)^{D}\right)^{2}\left(x_{1} \otimes x_{2} \otimes \ldots \otimes x_{n}\right)
$$

$=\left(T_{1}^{* 2} \otimes T_{2}^{* 2} \otimes \ldots \otimes T_{n}^{* 2}\right)\left(\left(T_{1}^{D}\right)^{2} \otimes\left(T_{2}^{D}\right)^{2} \otimes \ldots \otimes\left(T_{n}^{D}\right)^{2}\right)\left(x_{1} \otimes x_{2} \otimes \ldots \otimes x_{n}\right)$

$=T_{1}^{* 2}\left(T_{1}^{D}\right)^{2}\left(x_{1}\right) \otimes T_{2}^{* 2}\left(T_{2}^{D}\right)^{2}\left(x_{2}\right) \otimes \ldots \otimes T_{n}^{* 2}\left(T_{n}^{D}\right)^{2}\left(x_{n}\right)$

$=\left(T_{1}^{*} T_{1}^{D}\right)^{2}\left(x_{1}\right) \otimes\left(T_{2}^{*} T_{2}^{D}\right)^{2}\left(x_{2}\right) \otimes \ldots \otimes\left(T_{n}^{*} T_{n}^{D}\right)^{2}\left(x_{n}\right)$

$=\left(T_{1}^{*} T_{1}^{D}\left(x_{1}\right) \otimes T_{2}^{*} T_{2}^{D}\left(x_{2}\right) \otimes \ldots \otimes T_{n}^{*} T_{n}^{D}\left(x_{n}\right)\right)^{2}$

$=\left(\left(T_{1}^{*} \otimes T_{2}^{*} \otimes \ldots \otimes T_{n}^{*}\right)\left(T_{1}^{D} \otimes T_{2}^{D} \otimes \ldots \otimes T_{n}^{D}\right)\right)^{2}\left(x_{1} \otimes x_{2} \otimes \ldots \otimes x_{n}\right)$

$=\left(\left(T_{1} \otimes T_{2} \otimes \ldots \otimes T_{n}\right)^{*}\left(T_{1} \otimes T_{2} \otimes \ldots \otimes T_{n}\right)^{D}\right)^{2}\left(x_{1} \otimes x_{2} \otimes \ldots \otimes x_{n}\right)$

In the following theorem, we compute the Drazin invertible operator for some special matrix.

\section{Theorem 2.9:}

Let $T=\left(\begin{array}{ll}a & c \\ 0 & b\end{array}\right)$, where $a, b, c$ are non-zero complex numbers such that $a \neq b$, then one of the following forms of Drazin invertible can be satisfied:
1) $T^{D}=0$.
2) $T^{D}=\left(\begin{array}{cc}0 & -c /((a-b) b) \\ 0 & 1 / b\end{array}\right)$.
3) $\quad T^{D}=\left(\begin{array}{cc}1 / a & c /((a-b) a) \\ 0 & 0\end{array}\right)$.
4) $T^{D}=\left(\begin{array}{cc}1 / a & -c /(a b) \\ 0 & 1 / b\end{array}\right)$. 


\section{Proof:}

Let $T^{D}=\left(\begin{array}{ll}m_{1} & m_{2} \\ m_{3} & m_{4}\end{array}\right)$, where $m_{1}, m_{2}, m_{3}, m_{4} \in \mathbb{C}$, then

$$
\begin{gathered}
T T^{D}=T^{D} T \\
T^{D} T T^{D}=T^{D}
\end{gathered}
$$

Thus, from eq (1), it is easy to conclude that

$$
\left(\begin{array}{cc}
a m_{1}+c m_{3} & a m_{2}+c m_{4} \\
b m_{3} & b m_{4}
\end{array}\right)=\left(\begin{array}{ll}
a m_{1} & c m_{1}+b m_{2} \\
a m_{3} & c m_{3}+b m_{4}
\end{array}\right) \text {. }
$$

Therefore we get that

$$
\begin{aligned}
& a m_{1}+c m_{3}=a m_{1} \\
& a m_{2}+c m_{4}=c m_{1}+b m_{2}
\end{aligned}
$$

From (3), we get $m_{3}=0$.

This implies that, from eq (2), the following matrix equation:

$$
\left(\begin{array}{cc}
a m_{1}^{2} & a m_{1} m_{2}+m_{4}\left(c m_{1}+b m_{2}\right) \\
0 & b m_{4}^{2}
\end{array}\right)=\left(\begin{array}{ll}
m_{1} & m_{2} \\
m_{3} & m_{4}
\end{array}\right) \text {. }
$$

Thus we get the following equations:

$$
\begin{aligned}
& m_{1}\left(a m_{1}-1\right)=0 \\
& m_{4}\left(b m_{4}-1\right)=0 \\
& a m_{1} m_{2}+m_{4}\left(c m_{1}+b m_{2}\right)=m_{2}
\end{aligned}
$$

Thus, from equations (5), (6) and (7), we obtain the following cases:

Case 1: If $m_{1}=m_{4}=0$, then we have from eq (7) that $m_{2}=0$. Thus $T^{D}$ is the zero matrix.

Case 2: If $m_{1}=0, m_{4}=\frac{1}{b}$, then we have from eq (4) that $m_{2}=-c /((a-b) b)$. Thus $T^{D}=$ $\left(\begin{array}{cc}0 & -c /((a-b) b) \\ 0 & 1 / b\end{array}\right)$.

Case 3: If $m_{1}=1 / a, m_{4}=0$, then we have from eq (4) that $m_{2}=c /((a-b) a)$. Thus $T^{D}=$ $\left(\begin{array}{cc}1 / a & c /((a-b) a) \\ 0 & 0\end{array}\right)$.

Case 4: If $m_{1}=1 / a, m_{4}=1 / b$, then we have from eq (4) that $m_{2}=-c /(a b)$. Thus $T^{D}=$ $\left(\begin{array}{cc}1 / a & -c /(a b) \\ 0 & 1 / b\end{array}\right)$

\section{Remark 2.10:}

Note that, from theorem (2.9), the case (1) satisfies when $T$ is nilpotent matrix $\left(T^{D}=0\right)$ and case (4) satisfies when $T$ is invertible $\left(T^{D}=T^{-1}\right)$.

\section{Corollary 2.11:}

Let $T=\left(\begin{array}{ll}a & c \\ 0 & b\end{array}\right)$, where $a, b, c$ are non-zero complex numbers such that $a \neq b$. If $T^{D} \neq 0$, then $\underline{T}$ is not D-operator.

\section{Proof:}

We discuss case (2) in theorem (2.8) and the other cases can be proved similarly. Note that $T=\left(\begin{array}{ll}a & c \\ 0 & b\end{array}\right), T^{*}=\left(\begin{array}{ll}a & 0 \\ c & b\end{array}\right)$ and $T^{D}=\left(\begin{array}{cc}0 & -c /((a-b) b) \\ 0 & 1 / b\end{array}\right)$.

\section{Hence}

$T^{* 2}=\left(\begin{array}{cc}a^{2} & 0 \\ c(a+b) & b^{2}\end{array}\right)$ and $\left(T^{D}\right)^{2}=\left(\begin{array}{cc}0 & -c /\left((a-b) b^{2}\right) \\ 0 & 1 / b^{2}\end{array}\right)$

Thus

On the other hand,

$$
T^{* 2}\left(T^{D}\right)^{2}=\left(\begin{array}{cc}
0 & -c a^{2} /\left((a-b) b^{2}\right) \\
0 & \left(-c^{2}(a+b) /\left((a-b) b^{2}\right)\right)+1
\end{array}\right)
$$

$$
\left(T^{*} T^{D}\right)^{2}=\left(\begin{array}{cc}
-c^{2} a /\left((a-b) b^{2}\right) & -c a /((a-b) b) \\
c / b & \left(-c^{2} a /\left((a-b) b^{2}\right)\right)+1
\end{array}\right)
$$

Assume that $T$ is D-operator, then $T^{* 2}\left(T^{D}\right)^{2}=\left(T^{*} T^{D}\right)^{2}$. Therefore, from eqs (8) and (9), we obtain that $c / b=0$. Since $b \neq 0$, then $c=0$, which is a contradiction. Hence $M$ cannot be D-operator 


\section{Conclusions}

The present paper discusses some elementary properties of a new class of operators, namely the Doperators. The D-operators is some generalization of normal operators. Some properties of normal operator may not be satisfied in D-operators, such as the property of the sum and the product of two D-operators, which we proved that it is not necessarily true.

\section{References}

1. Caradus, S. R. 2004. Operator theory of the generalized inverse, Science Press, New York.

2. Ben-Israel, A. and Greville, T. N. 2003. Generalized inverses: Theory and applications, sec. ed., Springer Vralg, New York.

3. Campbell, S. R. and Meyer, C. D. 1991. Generalized inverses of linear transformations, Pitman, New York.

4. Jibril, A. A. S. 2010. On Operators for which $T^{* 2}(T)^{2}=\left(T^{*} T\right)^{2}$, International Mathematical Forum, 5(46): 2255 - 2262.

5. Dana, M. and Yousefi, R. 2018. On the classes of D-normal operators and D-quasi normal operators on Hilbert space, Operators and Matrices, 12(2): 465-487. 